\title{
Incidencia de la experiencia docente con el TDAH en los conocimientos, la autoeficacia y los niveles de estrés docente *
}

\section{Impact of teaching experience with ADHD on knowledge, self- efficacy and teacher stress}

Recepción: 06 Octubre 2017 | Aceptación: 16 Octubre 2019

\author{
MAnuel Soriano-Ferrer ${ }^{a}$ \\ Universidad de Valencia, España \\ ORCID: http://orcid.org/0000-0002-5836-4697 \\ JOYCE ECHEGARAY-BENGOA \\ Universidad de Valencia, España \\ ORCID: http://orcid.org/0000-0002-9658-7170
}

a Autor de correspondencia. Correo electrónico: manuel.soriano@uv.es

Para citar este artículo: Soriano-Ferrer, M., \& Echegaray-Bengoa, J. (2019). Incidencia de la experiencia docente con el TDAH en los conocimientos, la autoeficacia y los niveles de estrés docente. Universitas Psychologica, 18(4), 1-13. https:// doi.org/10.11144/Javeriana.upsy18-4.iedt

\section{RESUMEN}

Los estudios que han comparado los conocimientos que tienen los profesores sin experiencia (PSE) y los profesores con experiencia profesional (PCE) sobre el Trastorno por Déficit de Atención e Hiperactividad (TDAH) han mostrado resultados contradictorios. Además, el papel que tiene la experiencia docente con niños con TDAH sobre los conocimientos, la autoeficacia y el estrés docente no ha sido abordado en los estudios previos. Por ello, el presente estudio pretende: a) comparar los conocimientos, las creencias erróneas y las lagunas de conocimiento de los PCE y PSE de España, acerca del TDAH; b) analizar la experiencia de los PCE con niños con TDAH y la relación con los conocimientos, la autoeficacia, y el estrés docente; c) analizar las relaciones de los conocimientos acerca del TDAH con las variables sociodemográficas. Participaron 134 PSE y 115 PCE. Cumplimentaron la Escala de Conocimientos sobre el Trastorno por Déficit de Atención con Hiperactividad (Kadds), el Índice de Estrés Docente (ITS) y un cuestionario de información sociodemográfica. Los resultados indican que los conocimientos de ambos grupos son similares, aunque los PSE obtuvieron más aciertos sobre síntomas/diagnóstico y los PCE presentaron más creencias erróneas en la escala total y sobre síntomas/diagnóstico. Los PCE que tuvieron experiencia con niños con TDAH experimentaron mayor nivel de estrés docente y mayor autoeficacia. Los conocimientos sobre el TDAH correlacionaron con las asignaturas de carrera, los cursos sobre TDAH y los años de experiencia profesional.

\section{Palabras clave}

conocimientos; TDAH; creencias erróneas; profesores; autoeficacia; estrés docente.

\begin{abstract}
Studies that have compared the knowledge that pre-service teachers (PSE) and in-service teachers (PCE) have about ADHD have shown conflicting results. In addition, the role that teaching experience with children with ADHD has on knowledge, self-efficacy and teacher stress has not been addressed in previous studies. Therefore, this study aims to: a) compare the knowledge, misconceptions and knowledge gaps of
\end{abstract}


the PCE and PSE of Spain, about ADHD; b) analyze the experience of PCE with children with ADHD and the relationship with knowledge, self-efficacy, and teacher stress; c) analyze the relations of knowledge about ADHD with sociodemographic variables. 134 PSE and 115 PCE participated. They completed the Knowledge Scale on Attention Deficit Hyperactivity Disorder (KADDS), the Teaching Stress Index (STI) and a socio-demographic information questionnaire. The results indicate that the knowledge of both groups is similar, although the PSE obtained more successes on symptoms / diagnosis and the PCE presented more misconceptions on the total scale and on symptoms / diagnosis. The PCE, who had experience with children with ADHD, experienced a higher level of teacher stress and greater self-efficacy. Knowledge about ADHD correlated with career subjects, courses on ADHD and years of professional experience.

Keywords

knowledge; ADHD; misconceptions; teachers; self-efficacy; teacher stress.

\section{El Trastorno por Déficit de Atención e Hiperactividad (TDAH) afecta al} neurodesarrollo, y se caracteriza por presentar un patrón persistente de conductas de inatención, exceso de actividad y dificultad para controlar los impulsos, que influyen negativamente en el desarrollo social, afectivo, e incluso en el aprendizaje (American Psychiatric Association, 2014; Hinshaw, 2018). Los estudios señalan una prevalencia que oscila entre el 1-20\%, lo que posiblemente se debe a aspectos metodológicos, demográficos y culturales, tal como se destaca en un reciente meta-análisis (Polanczyk, Salum, Sugaya, Caye, \& Rohde, 2015). En concreto, en España, a partir de un estudio de revisión meta-analítica (Catalá-López et al., 2012), se concluye que la prevalencia es de $6.8 \%$, aunque en edad preescolar es del $5.4 \%$ (Canals, Morales-Hidalgo, Jané, \& Domènech, 2018). Sin embargo, a pesar de esta alta prevalencia del TDAH, que sugiere que es muy probable que cada profesor tenga en el aula, al menos a un alumno con TDAH, los estudios que han analizado los conocimientos que tienen los profesores acerca del TDAH han señalado que los porcentajes de aciertos de los maestros oscilan entre bajos a moderados. En concreto, algunos estudios que han empleado el mismo instrumento de evaluación de los conocimientos acerca del TDAH que en este estudio han arrojado porcentajes de aciertos muy dispares. Así, a modo ilustrativo, el $17.2 \%$ acertaron en el estudio de Alkahtani (2013), el $42.65 \%$ en los estudios de Jarque, Tárraga y Miranda (2007) y Jarque y Tárraga (2009), y el 60.7 $\%$ acertaron en el estudio de Kos, Richdale y Jackson (2004). Esta falta de conocimientos de los profesores sobre el TDAH, así como el propio comportamiento de los alumnos con TDAH, afectan negativamente al proceso de enseñanzaaprendizaje (Anderson, Watt, \& Shanley, 2017; Lawrence, Estrada, \& McCormick, 2018; Rogers \& Tannock, 2018; Toye, Wilson, \& Wardle, 2019), lo que incrementa el estrés de los profesores (Choi, 2017; Greene, Abidin, \& Kmetz, 1997; Raggi, Evans, Hackethorn, \& Thompson, 2003; Skaalvik \& Skaalvik, 2009).

Aunque durante las dos últimas décadas se han realizado una gran cantidad de estudios sobre los conocimientos de los profesores acerca del TDAH, tanto en América del Norte (Canu, \& Mancil, 2012; Graczyk et al., 2005; Jerome, Gordon, \& Hustler, 1994; Piccolo-Torsky \& Waishwell, 1998; Hepperlen, Clay, Henly, \& Barké, 2002; Poznanski, Hart, \& Cramer, 2018; Snider, Busch, \& Arrowood, 2003), como en Australia, Europa y Asia (Alkahtani, 2013; Akram, Thomson, Boyter, \& McLarty, 2009; Bekle, 2004; Brook, Watemberg, \& Geva, 2000; Ghanizadeh, Bahredar, \& Moeini, 2006; Havey, 2007; Holst, 2008; Hong, 2008; Jarque \& Tárraga, 2009; Jarque et al., 2007; Kos et al., 2004; Nur \& Kavakci, 2010; Ohan, Cormier, Hepp, Visser, \& Strain, 2008; Soroa, Gorostiaga, \& Balluerka, 2016; Syed \& Hussein, 2010; West, Taylor, Houghton, \& Hudyma, 2005), e incluso se han comparado los conocimientos de los profesores entre países (Jerome et al., 1994; Norvilitis \& Fang, 2005; Sciutto et al., 2016). Los estudios que han comparado los conocimientos que tienen los profesores sin experiencia (PSE) y los profesores con experiencia profesional (PCE) sobre el TDAH son muy escasos y muestran resultados contradictorios (Anderson, Watt, Noble, \& Shanley, 2012; Akram et al., 2009; Bekle, 2004; Jarque \& Tárraga, 2009; Jerome, Washington, Laine, \& Segal, 1999; Kos et al., 
2004). Por un lado, algunos estudios indican que los conocimientos de los PCE son superiores a los de los PSE o bien, que los conocimientos de ambos grupos de profesores son similares. Por ejemplo, en Australia, Kos et al. (2004) pusieron de manifiesto que los conocimientos de los PCE $(60.7 \%)$ eran significativamente superiores a los de los PSE (52.6 \%). También en Australia, el estudio de Anderson et al. (2012) señala que los conocimientos de los PCE (60.2 \%) son mayores a los de los PSE (52.9\%). Por otro lado, algunos estudios muestran que los conocimientos de los PSE son similares a los de los PCE, e incluso ligeramente superiores. Así, el estudio de Jerome et al. (1999), realizado en EE. UU, puso en relieve que los conocimientos acerca del TDAH eran similares en los PSE y los PCE (77 y $78 \%$, respectivamente). En la misma línea, el estudio de Bekle (2004) en Australia evidenció un nivel de conocimientos similar en ambos grupos de profesores: $82 \%$ en los PCE y $75 \%$ en los PSE. En Escocia, el estudio de Akram et al. (2009) mostró que, aunque los conocimientos eran escasos en ambos grupos, los PSE sabían más sobre el tratamiento farmacológico del TDAH que los PCE. En España, Jarque y Tárraga (2009) no encontraron diferencias significativas entre los conocimientos de los PCE (42.6\%) y los PSE (43.6\%).

Muchos estudios han tratado de relacionar los conocimientos sobre el TDAH y la experiencia profesional, específicamente la experiencia directa con alumnos con este trastorno. De hecho, son numerosos los estudios que muestran que los conocimientos acerca del TDAH de los profesores correlacionaban positivamente con los años de experiencia profesional y/o con la experiencia previa con niños con TDAH (Anderson et al., 2012; Bekle, 2004; Jerome et al., 1994; Jerome et al., 1999; Kos et al., 2004; Sciutto, Terjesen, \& Bender Frank, 2000; Sciutto et al., 2016; Soroa et al., 2016; Weyandt, Fulton, Schepman, Verdi, \& Wilson, 2009).

También diferentes estudios han relacionado los conocimientos acerca del TDAH con los sentimientos de autoeficacia a la hora de enseñar a un niño con este trastorno, es decir, que los profesores que se sienten más competentes tienen más conocimientos acerca del TDAH (Alkahtani, 2013; Blotnicky-Gallant, Martin, McGonnell, \& Corkum, 2015; Jarque et al., 2007; Soroa et al., 2016; Sciutto et al., 2000). Sin embargo, otros estudios no han encontrado una correlación significativa entre los conocimientos totales sobre TDAH y sus sentimientos de autoeficacia en el trabajo con niños con TDAH (Graeper, Barker, \& Terjesen, 2008; Jarque \& Tárraga, 2009; Poznanski, Hart, \& Cramer, 2018).

Por ello, partiendo de la panorámica que muestra la investigación previa, en el presente estudio nos planteamos diferentes objetivos. En primer lugar, pretendemos comparar los conocimientos, las creencias erróneas y lagunas de conocimiento de los PCE y de los PSE acerca del TDAH, ya que, como hemos visto, los estudios previos han mostrado resultados contradictorios. En segundo lugar, se pretende analizar el papel que tiene la experiencia en la educación de niños con TDAH en los conocimientos que tienen los PCE, los sentimientos de autoeficacia, así como en los niveles de estrés docente, ya que ningún estudio ha comprobado si existen diferencias estadísticamente significativas en los conocimientos entre los PCE, con y sin experiencia docente con niños con TDAH, ya que todos han empleado exclusivamente análisis correlacionales. Por último, nuestro tercer objetivo se dirige al análisis de las relaciones que tienen los conocimientos acerca del TDAH y diferentes variables sociodemográficas.

\section{Método}

\section{Participantes}

La muestra estaba compuesta por 249 profesores en total, de los que 134 eran profesores sin experiencia (PSE) y 115 eran profesores con experiencia (PCE). Todos los participantes son de la Comunidad Valenciana. Los PSE proceden de dos universidades públicas y los PCE trabajaban en centros públicos de dos provincias de la Comunidad Valenciana. De los PSE, 113 eran 
mujeres (97 \%) y 4 fueron hombres (3\%), mientras que, en el grupo de los PCE, 90 fueron mujeres (78.3 \%) y 25 hombres (21.7 \%). La edad promedio de los PSE era de 24 años y 2 meses $(D T=5.9)$, mientras que la edad promedio de los PCE fue de 38 años y 4 meses (DT $=8.5$ ). El promedio de la experiencia enseñando de los PCE fue de entre 1 y 10 años. De los PSE, 86 se preparaban para ser maestros de educación infantil, y 48 de enseñanza primaria. De los PCE, 32 eran maestros de infantil, 75 de primaria, y 8 de secundaria. Además, 15 de los PCE (13\%) tenían un título de postgrado (ver Tabla 1).

Como era de esperar, los resultados de los Anova señalan que los PSE y los PCE difieren significativamente en cuanto a la edad cronológica, $\left.F_{(1,247}\right)=238.71, p<0.001, \eta^{2}$ $=0.49$, y en cuanto a la experiencia profesional, $F_{(1,247)}=496.327, p<0.001, \eta^{2}=0.66$, con un tamaño del efecto grande, que es mayor en el caso de los PCE. En cuanto a los sentimientos de autoeficacia, también son los PCE los que reportan mayores niveles a la hora de trabajar con alumnos con TDAH, $F_{(1,247)}=5.303, p<$ $0.01, \eta^{2}=0.02$, aunque con un tamaño del efecto pequeño.

Respecto a la formación previa sobre el TDAH, el $22.4 \%(n=30)$ de los PSE había tenido contacto con niños con este trastorno durante los meses de prácticas en centros educativos. En el caso de los PCE, el $37.4 \%(n=43)$ trabajó con algún niño con TDAH durante, al menos, un curso escolar, mientras que $62.6 \%(n=72)$ no habían tenido experiencia profesional directa de trabajo con niños con TDAH. Todos los niños con TDAH de los centros educativos cuentan con un diagnóstico realizado por los Equipos Psicopedagógicos Escolares.

Respecto a las fuentes de información sobre el TDAH empleadas, el 81.3 \% informó que había cursado alguna asignatura de carrera que trataba el TDAH, el $25.4 \%$ había realizado algún curso o asistido a alguna charla sobre el TDAH, y el 21.6 \% había visto u oído algún programa de TV/radio sobre el TDAH. En el caso de los PCE, el 90.4 $\%$ accedía a la información acerca del TDAH a través de programas de TV/radio, el $59.1 \%$ a través de libros especializados, el 54.8 \% había conseguido información acerca de las asignaturas de carrera, y el $48.7 \%$ a través de cursos o charlas especializadas.

Por su parte, los resultados de los Anova señalan que los $\mathrm{PCE}$, con y sin experiencia profesional con alumnos con TDAH, no difieren en cuanto a la edad cronológica, $\left.F_{(1,113}\right)=$ 1864, $p<0.175, \eta^{2}=0$, ni en relación a los años de experiencia profesional, $F_{(1,113}$ ) $=0.167, p<0.684, \eta^{2}=0$. Sin embargo, los PCE con alumnos con TDAH tienen mayores sentimientos de autoeficacia a la hora de trabajar con alumnos con este trastorno, $\left.F_{(1,113}\right)=$ 13.132, $p<0.001, \eta^{2}=0.1$, con un tamaño del efecto mediano, y experimentan mayores niveles de estrés docente, $F_{(1,113)}=5.341, p<0.01, \eta^{2}$ $=0.08$, con un tamaño del efecto pequeño.

\section{Tabla 1}

Información Demográfica de la muestra de profesores con y sin experiencia

\begin{tabular}{|c|c|c|c|c|}
\hline \multirow{2}{*}{ Caracteristicas } & \multicolumn{2}{|c|}{ PSE } & \multicolumn{2}{|c|}{ PCE } \\
\hline & $N=134$ & $\%$ & $N=115$ & $\%$ \\
\hline \multicolumn{5}{|l|}{ Género } \\
\hline Mujer & 130 & 97 & 90 & 78.3 \\
\hline Hombre & 4 & 3 & 25 & 21.7 \\
\hline \multicolumn{5}{|l|}{ Edad } \\
\hline $\mathrm{M}$ & 24.2 & & 38.4 & \\
\hline DT & 5.9 & & 8.5 & \\
\hline \multicolumn{5}{|l|}{ Experiencia enseñando en años } \\
\hline 0 & 134 & 100 & 0 & 0 \\
\hline $1-10$ & 0 & 0 & 46 & 40 \\
\hline $11-20$ & 0 & 0 & 39 & 33.9 \\
\hline $21+$ & 0 & 0 & 30 & 26.1 \\
\hline \multicolumn{5}{|l|}{ Nivel que enseña } \\
\hline Infantil & 86 & 64.2 & 32 & 27.8 \\
\hline Primaria & 48 & 35.8 & 75 & 65.2 \\
\hline Secundaria & 0 & 0 & 8 & 7 \\
\hline \multicolumn{5}{|l|}{ Educación del profesor } \\
\hline Grado & 134 & 100 & 115 & 100 \\
\hline Posgrado & 0 & 0 & 15 & 13 \\
\hline \multicolumn{5}{|c|}{ Experiencia con niño con $T D A H$} \\
\hline $\mathrm{Si}$ & 30 & 22.4 & 43 & 37.4 \\
\hline Algunos meses & 30 & 22.4 & 0 & 0 \\
\hline 1 año & 1 & 0.9 & 24 & 20.9 \\
\hline 2 años & 0 & 0 & 9 & 7.8 \\
\hline $3+$ & 0 & 0 & 10 & 8.7 \\
\hline \multicolumn{5}{|l|}{ Fuentes de información ${ }^{a}$} \\
\hline Asignatura de carrera & 109 & 81.3 & 63 & 54.8 \\
\hline Libros & 17 & 12.7 & 68 & 59.1 \\
\hline TV/radio & 29 & 21.6 & 104 & 90.4 \\
\hline Cursos especiales/charlas & 34 & 25.4 & 59 & 48.7 \\
\hline Revistas especializadas & 4 & 3 & 11 & 9.6 \\
\hline Ninguna & 25 & 78.4 & 4 & 3.5 \\
\hline \multicolumn{5}{|l|}{ Autoeficacia } \\
\hline M & 2.76 & & 3.05 & \\
\hline DT & 1.11 & & 0.83 & \\
\hline
\end{tabular}

Notas ${ }^{a}$ supera el $100 \%$ debido a que los maestros podían seleccionar más de una fuente de información 
Instrumentos

Escala de Conocimientos sobre los Trastornos por Déficit de Atención con Hiperactividad (Knowledge of Attention Deficit Disorders Scale Kadds, Sciutto et al., 2000)

Está compuesta por 36 ítems que se agrupan en tres subescalas: a) información general sobre la naturaleza, causas y repercusiones del TDAH (15 ítems); b) síntomas/diagnóstico de TDAH (9 ítems); y c) tratamiento del TDAH (12 ítems). El formato de tres opciones de respuesta de este cuestionario permite conocer, no solo los conocimientos (aciertos) sobre el trastorno, sino también las concepciones erróneas (errores), y las lagunas (dudas). Es el instrumento más empleado en la mayoría de los estudios sobre el tema. La adaptación española del Kadds ha mostrado tener elevados índices de fiabilidad, obteniéndose coeficientes alfa, que oscilan entre el 0.87 y el 0.89 (Jarque et al., 2007).

Índice de Estrés Docente (Index of Teaching StressITS) (Greene et al., 1997)

Se trata de una escala de 90 ítems tipo Likert de cinco puntos, que evalúa el nivel de angustia docente en relación con un niño en particular de su aula. La puntuación es un indicador de tensión total de las experiencias de los maestros con experiencia profesional con relación a un estudiante determinado, por lo que solo fue cumplimentado por los PCE.

\section{Cuestionario de Información Sociodemográfica}

Se diseñó un cuestionario ad hoc para el estudio, en el que los participantes dieron información sobre su edad, género, años de experiencia profesional, formación profesional, medios de información a los que acceden, y si habían tenido experiencia enseñando a niños con TDAH. Además, incluía una escala Likert de siete puntos (teniendo en cuenta que 1 significa no estar preparado y 7 significa estar totalmente preparado), donde se preguntaba a los profesores acerca de sus sentimientos de autoeficacia para enseñar a niños con TDAH, "ihasta qué punto consideras que puedes enseñar de forma efectiva a un niño con TDAH?".

\section{Procedimiento}

Los PSE cumplimentaron los cuestionarios durante el último año de formación profesional en sus universidades, dos de carácter público de la Comunidad Valenciana, después de su último periodo de prácticas preprofesionales. Se excluyeron a aquellos que por sus calificaciones no obtenían su título de graduado. Los PCE cumplimentaron los cuestionarios en los colegios donde ejercían profesionalmente, todos ellos colegios públicos de dos provincias de la Comunidad Valenciana, en presencia de uno de los autores para aclarar cualquier posible duda. Todos los profesores participaron de forma anónima, voluntaria y desinteresada en este estudio.

\section{Análisis de datos}

Todos los análisis estadísticos fueron realizados mediante el programa informatizado SPSS, versión 22. Se comprobó que los datos cumplían el criterio de normalidad estadística, aplicando la prueba de Kolmogorov-Smirnov. En aquellos casos en los que la distribución era normal $(p>0.05)$, se realizaron análisis de varianza (Anova) de comparación entre grupos, mientras que el test U de Mann-Whitney fue empleado en caso contrario. Para efectos de interpretación, se aplicó la corrección de Bonferroni para determinar los niveles de significación que se considerarían como relevantes. Además, se aportó el tamaño del efecto mediante la eta cuadrado $\left(\eta^{2}\right)$ en el caso de los análisis paramétricos (valores entre 0.01 y 0.1 se consideran efecto de tamaño pequeño, entre 0.1 y 0.3 , como efecto mediano, y valores superiores a 0.3 como efectos grandes) y la $r$ en el caso de las no paramétricas (valores inferiores a $0.2 \mathrm{se}$ 
consideran como tamaño del efecto de pequeño, 0.3 de magnitud media y 0.5 como tamaño del efecto grande).

Con el fin de relacionar los conocimientos de los PSE y PCE con las diferentes variables sociodemográficas, se realizaron análisis correlacionales.

Comparación entre los profesores sin experiencia (PSE) y los profesores con experiencia (PCE)

En relación con los aciertos (véase la Tabla 2), el test $U$ de Mann-Whitney indica que no se encuentran diferencias significativas entre los PSE y los PCE en las subescalas de información general, $U_{(248)}=7299, Z=-0.723, p<0.46$, $r=0.04$, tratamiento, $U_{(248)}=7441.5, Z=$ -0.47, $p<0.63, r=0.03$, ni en la escala total, $U_{(248)}=7082.5, Z=-1.101, p<0.27, r=$ 0.06. Sin embargo, en la subescala de síntomas/ diagnóstico, los PSE tuvieron significativamente más aciertos que los PCE, (RP PSE $=141.95 \mathrm{y}$ $\mathrm{RP} P C E=105.25), U_{(248)}=5434, Z=-4.066$, $p<0.001, r=0.25$.

En un análisis individualizado de los ítems, se encontraron algunas diferencias significativas entre los PSE y los PCE. En concreto, los PCE tuvieron más aciertos que los PSE en dos ítems pertenecientes a la subescala de tratamiento. Específicamente, los PCE conocen de forma más significativa que "las intervenciones conductuales/psicológicas para los niños con TDAH se centran principalmente en los problemas del niño con la falta de atención" (ítem 34), $U$ (248) $=6061, Z=$ -3.543, $p<0.001, r=0.22$, así como que "los fármacos estimulantes son el tipo más común de medicamentos utilizados para tratar a los niños con TDAH" (ítem 25), $U_{(248)}=5941, Z$ $=-3.742, p<0.001, r=0.24$. Por su parte, los PSE sabían que no es cierto que "reducir la ingesta de azúcar o aditivos alimentarios es generalmente eficaz en la disminución de los síntomas de TDAH" (ítem 23), $U_{(248)}=6266.5$, $Z=-3.632, p<0.001, r=0.23$.

Entre los ítems con más aciertos del estudio, tanto en los PSE como en los PCE (véase Tabla
3), se aprecia que la mayoría de los profesores (90.3 \% de los PSE y $80.9 \%$ de los PCE) sabe que los chicos con TDAH se distraen frecuentemente por estímulos externos, que tienen dificultades para organizar tareas y actividades $(88.8 \%$ de los PSE y $78.3 \%$ de los PCE), que se inquietan o se retuercen en sus asientos $(85.1 \%$ de los PSE y $73.9 \%$ de los PCE), que los síntomas deben estar presentes en, al menos, dos entornos (83.6 \% de los PSE y $74.8 \%$ de los PCE), y que los tratamientos basados en el castigo no son efectivos para la reducción de los síntomas de TDAH (80.6 \% de los PSE y $75.7 \%$ de los PCE).

\section{Tabla 2}

Estadísticos descriptivos de los PSE y los PCE en cada una de las subescalas

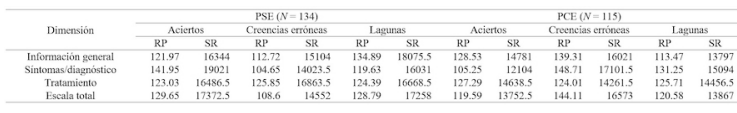

NotaRP $=$ rango promedio, $\mathrm{SR}=$ suma de rangos

Con relación a las creencias erróneas (véase la Tabla 2), el test U de Mann-Whitney indica que hay diferencias significativas en la escala total, $U_{(248)}=5507, Z=-3.899, p<0.001, r=$ 0.25 , entre los PSE y los PCE, donde los últimos tuvieron mayores creencias erróneas (RP PCE $=$ 144.11 y RP PSE $=108.6$ ). En la misma línea, también los PCE tuvieron significativamente más creencias erróneas que los PSE en las subescalas de información general (RP PCE $=139.31$ y RP $\mathrm{PSE}=112.72), U_{(248)}=6059, Z=-2.933, p$ $<0.003, r=0.19$, de síntomas/diagnóstico (RP $\mathrm{PCE}=148.71$ y $\mathrm{RP} P S E=104.65), U_{(248)}=$ $4978.5, Z=-5.119, p<0, r=0.32$. Sin embargo, en la subescala de tratamiento no se encontraron diferencias significativas, $U_{(248)}=7591.5, Z=$ $-0.209, p<0.83, r=0.01$.

En el análisis individualizado de los ítems, los PCE tuvieron significativamente mayores creencias erróneas en tres ítems. Por ejemplo, creen en mayor medida que "la prevalencia del TDAH en función del género es equivalente en niños y niñas" (ítem 29), $U$ (248) $=6567, Z=$ -3.068, $p<0.002, r=0.19$, que "los niños con TDAH tienen un sentido de grandiosidad o una autoestima inflada" (ítem 11), $U_{(248)}=6480.5$, $Z=-3.59, p<0.001, r=0.23$, y que, "el TDAH 
es debido a las deficientes habilidades parentales de sus padres" (ítem 2), $U_{(248)}=6671.5, Z=$ -3.79, $p<0.001, r=0.24$. Sin embargo, son los PSE los que creen que "los antidepresivos no han sido eficaces para reducir los síntomas de los niños con TDAH" (ítem 8), $U_{(248)}=5873, Z=$ $-4.101, p<0.001, r=0.26$.

En ambos grupos de profesores (PSE y PCE), las creencias erróneas más frecuentes (véase Tabla 3) han sido que los médicos pueden hacer el diagnóstico basándose en características físicas (62.7\% de los PSE y $68.7 \%$ de los PCE), que deben recibir educación especial directamente por el hecho de ser TDAH $(39.6 \%$ de los PSE y $61.7 \%$ de los PCE), y que experimentan más dificultades en las situaciones novedosas $(50.7 \%$ de los PSE y $43.5 \%$ de los PCE). También creen erróneamente que en las situaciones de juego libre son más distinguibles los niños con TDAH que en el entorno de clase ( $53 \%$ de los PSE y $60.9 \%$ de los PCE), y que los niños con TDAH no suelen ser más obedientes con sus padres que con sus madres $(54.5 \%$ de los PSE y $60.9 \%$ de los PCE).

Por último, con relación a las lagunas de conocimiento (véase la Tabla 2), el test $U$ de Mann-Whitney indica que no se encontraron diferencias significativas entre los PSE y los PCE en ninguna de las subescalas: información general, $U_{(248)}=6379.5, Z=-2.349, p<0.01$, $r=0.15$, síntomas/diagnóstico, $U_{(248)}=6986$, $Z=-1.303, p<0.19, r=0.08$, tratamiento, $U_{(248)}=7623.5, Z=-0.145, p<0.884, r=$ 0.01 , ni en la escala total, $U_{(248)}=7197, Z=$ $-0.898, p<0.36, r=0.06$. Sin embargo, en el análisis individualizado de ítems, fueron los PSE los que desconocían significativamente que "la mayoría de los niños con TDAH presentan, en alguna medida, resultados académicos pobres en la etapa de primaria" (ítem 32), $U_{(248)}=6515$, $Z=-2.775, p<0.006, r=0.18$.

Como podemos apreciar en la Tabla 3, alrededor del $50 \%$ de los profesores desconocen la prevalencia del TDAH $(53.7 \%$ de los PSE y $59.1 \%$ de los PCE), la falta de eficacia de la terapia electroconvulsiva $(72.4 \%$ de los PSE y $80 \%$ de los PCE), y que el TDAH puede diagnosticarse en la edad adulta $(46.3 \%$ de los PSE y $50.9 \%$ de los PCE). Tampoco saben acerca de la mayor predisposición familiar del TDAH (67.2 \% de los PSE y $57.4 \%$ de los PCE), ni de la mayor vulnerabilidad de los niños con TDAH a experimentar sintomatología depresiva $(61.2 \%$ de los PSE y $60 \%$ de los PCE).

\section{Tabla 3}

Respuestas correctas, creencias erróneas y lagunas de conocimiento más comunes de los PSE y los

PCE

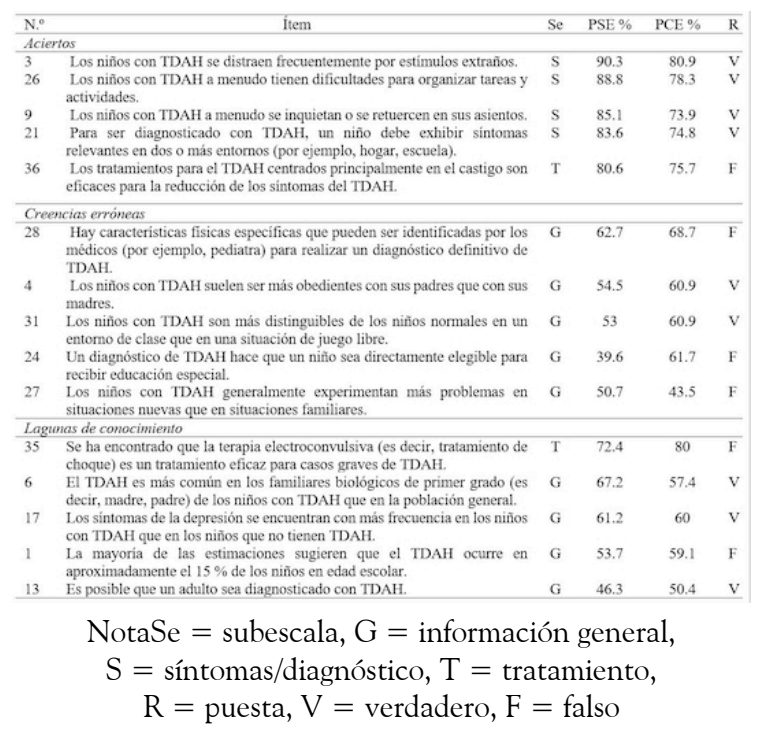

Comparación de los profesores con experiencia (PCE) con y sin experiencia profesional con alumnos con TDAH

Con relación a los aciertos (véase la Tabla 4), el test $U$ de Mann-Whitney indica que no se encuentran diferencias entre los PCE — con y sin experiencia en la enseñanza a niños con TDAH - en las subescalas de información general, $U$ $(113)=1482, Z=-0.385, p<0.7, r=0.03$, tratamiento, $U_{(113)}=1188.5, Z=-1.78, p$ $<0.07, r=0.16$, ni en el total de la escala, $U_{(113)}=1188.5, Z=-1.77, p<0.07, r=$ 0.16 . Sin embargo, en relación con los aciertos en las diferentes subescalas, aún sin alcanzar la significación estadística, la suma de rangos es ligeramente superior en el grupo de PCE con experiencia en la educación de niños con TDAH (véase Tabla 4). Por el contrario, en la 
subescala de síntomas se encuentran diferencias significativas entre los dos grupos de profesores, $U_{(113)}=1006, Z=-3.178, p<0.001, r=$ 0.31 , donde los profesores con experiencia en la educación de niños con TDAH son los que tienen un mayor número de aciertos.

\section{Tabla 4}

Estadísticos Descriptivos de los PCE con y sin experiencia profesional en TDAH

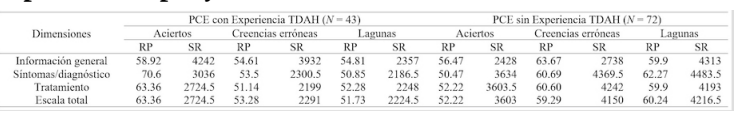

NotaRP $=$ rango promedio, $\mathrm{SR}=$ suma de rangos

En cuanto a las creencias erróneas (véase la Tabla 4), el estadístico U de Mann-Whitney tampoco señala diferencias significativas entre los PCE con y sin experiencia en niños con TDAH en ninguna de las subescalas: información general, $U_{(113)}=1304, Z=-1.437, p<0.15, r$ $=0.13$, síntomas $/$ diagnóstico, $U_{(113)}=1354, Z$ $=-1.168, p<0.24, r=0.1$, tratamiento, $U_{(113)}$ $=1253, Z=-1.556, p<0.12, r=0.14$, ni en la escala general, $U_{(113)}=1345, Z=-0.956, p$ $<0.33, r=0.08$. Aunque no existen diferencias significativas, los rangos promedios de creencias erróneas son ligeramente superiores en el caso de los PCE sin experiencia en la educación de niños con TDAH.

Por último, en relación con las lagunas de conocimiento (véase la Tabla 4), el estadístico U de Mann-Whitney tampoco señala diferencias significativas entre los PCE con y sin experiencia en niños con TDAH en ninguna de las subescalas: información general, $U(113)=1411$, $Z=-0.797, p<0.42, r=0.07$, síntomas $/$ diagnóstico, $U_{(113)}=1240, Z=-1.844, p<$ 0.06, $r=0.17$, tratamiento, $U_{(113)}=302, z$ $=-1.219, p<0.22, r=0.11$, ni en la escala general, $U_{(113)}=1278345, Z=-0.956, p<$ $0.33, r=0.08$. Aunque no existen diferencias significativas, los rangos promedios de lagunas de conocimiento son ligeramente superiores en el caso de los PCE sin experiencia en la educación de niños con TDAH.
Relación entre los conocimientos y las variables sociodemográficas

Los conocimientos de los maestros no correlacionaban con la edad cronológica. En el caso de los PSE, encontramos una correlación significativa entre los aciertos totales en la escala y las asignaturas sobre TDAH en sus estudios de universidad $(r=0.21, p<0.001)$, y con la autoeficacia percibida para enseñar a un niño con TDAH $(r=0.308, p<0.001)$. Por otro lado, en lo que concierne a los PCE, se encontró una correlación significativa entre el total de aciertos en la escala y los años de experiencia profesional $(r=0.323, p<0.001)$, así como con los cursos y charlas recibidas $(r=0.39, p$ $<0.001)$. Finalmente, también se encuentra una correlación significativa entre los conocimientos totales sobre TDAH y sus sentimientos de autoeficacia en el trabajo con niños con este trastorno $(r=0.274, p<0.001)$.

\section{Discusión}

Nuestro primer objetivo era comparar los conocimientos, las creencias erróneas y las lagunas de información de los PSE y los PCE con relación al TDAH. Básicamente, nuestros hallazgos son coincidentes con los de otros estudios previos que no han encontrado diferencias significativas entre los PSE y los PCE (Akram et al., 2009; Bekle, 2004; Jarque \& Tárraga, 2009; Jerome et al., 1999). De hecho, los aciertos totales de los PSE son del $57.5 \%$, y los de los PCE del $55.2 \%$. No obstante, en la escala de síntomas/diagnóstico, son los PSE los que tienen significativamente más aciertos que los PCE (55.3 \% PSE frente al $50.7 \%$ de los PCE). También son numerosos los estudios que han encontrado que los conocimientos relacionados con los síntomas/diagnóstico son más altos que los de las otras escalas (Blotnicky-Gallant et al., 2015; Guerra \& Brown, 2012; Jarque et al., 2007; Perold, Louw, \& Kleynhans, 2010; Sciutto et al., 2000).

En relación con nuestro segundo objetivo, analizar el rol que tiene la experiencia profesional 
con niños con TDAH en los conocimientos, los sentimientos de autoeficacia y los niveles de estrés docente, nuestros resultados son clarificadores. Los PCE con experiencia docente con niños con TDAH obtienen mayores aciertos en las diferentes escalas, muestran menos concepciones erróneas y menos lagunas de conocimiento que los PCE sin experiencia con niños con TDAH. Estos resultados son novedosos en el sentido en que es el primer estudio que muestra diferencias estadísticamente significativas en los conocimientos sobre el TDAH entre los PCE, con y sin experiencia docente con niños con TDAH. Si bien es cierto que numerosos estudios han correlacionado positivamente los mayores conocimientos sobre el TDAH con la experiencia docente, e incluso con la experiencia directa con niños con TDAH (Anderson et al., 2012; Bekle, 2004; Jerome et al., 1994; Jerome et al., 1999; Kos et al., 2004; Sciutto et al., 2000; Sciutto et al., 2016; Soroa et al., 2016; Weyandt et al., 2009), nuestro trabajo muestra que son los PCE que han tenido experiencia directa con un niño con TDAH, los que tienen mayores sentimientos de autoeficacia, aunque también son los que experimentan niveles más altos de estrés docente, tal y como han mostrado otros estudios (Choi, 2017; Greene et al., 1997; Skaalvik \& Skaalvik, 2009).

Por último, en cuanto a nuestro tercer objetivo, analizar las relaciones entre los conocimientos sobre el TDAH y diferentes variables sociodemográficas, nuestros datos muestran una correlación positiva entre los conocimientos sobre el TDAH y los sentimientos de autoeficacia, tanto en los PSE como en los PCE, al igual que en los estudios realizados previamente en diferentes países (Alkahtani, 2013; Blotnicky-Gallant et al., 2015; Jarque et al., 2007; Sciutto et al., 2000; Sciutto et al., 2016; Soroa et al., 2016; Weyandt et al., 2009), lo que no es extraño, ya que dichas variables sociodemográficas pueden constituir diferentes fuentes de información sobre el TDAH (Sciutto et al., 2016). Otro aspecto que merece ser destacado es que, al igual que otros muchos estudios (Anderson et al., 2012; Bekle, 2004;
Jerome et al., 1994; Jerome et al., 1999; Kos et al., 2004; Sciutto et al., 2000; Soroa et al., 2016; Weyandt et al., 2009), encontramos que los conocimientos acerca del TDAH estaban relacionados con las asignaturas de carrera sobre el TDAH en los PSE, y con los años de experiencia profesional y los cursos y las charlas recibidas en el caso de los PCE. Sin embargo, otros estudios no han encontrado relación entre los conocimientos sobre el TDAH y la experiencia profesional (Jarque \& Tárraga, 2009; Poznanski, Hart, \& Cramer, 2018).

Nuestros resultados contribuyen al conocimiento de la formación de los maestros, con y sin experiencia profesional, en torno al TDAH, así como a la influencia que algunas variables sociodemográficas tienen sobre dicha formación. No obstante, habría que referirse a una serie de limitaciones que pueden afectar el alcance de los hallazgos. La principal hace referencia a la selección de la muestra, de conveniencia, tomada exclusivamente de la Comunidad Valenciana, que hace que los resultados no se puedan generalizar al resto de España. Otra limitación tiene que ver con el hecho de que no se ha contemplado el expediente académico de los maestros, ya que es posible que aquellos profesores con calificaciones mayores en su expediente también tengan un mayor conocimiento sobre el TDAH.

Por otro lado, deben considerarse las implicaciones del uso del sistema de evaluación de los conocimientos empleado. Es decir, la evaluación de los conocimientos, creencias erróneas y lagunas de conocimiento mediante una escala de 36 ítems de respuesta cerrada solo proporciona una visión superficial de los conocimientos y creencias que tienen los maestros. De hecho, una puntuación alta en la escala no implica que los maestros dispongan de mayores habilidades y conocimientos para implementar programas de tratamiento con niños con TDAH.

No obstante, con independencia de las limitaciones apuntadas, nuestros hallazgos tienen importantes repercusiones educativas. Precisamente, este estudio ha proporcionado alguna información importante al diferenciar 
lo que los profesores no saben de lo que creen incorrectamente saber (es decir, sus creencias erróneas). Como afirmaba Sciutto et al. (2000), la distinción entre las creencias erróneas y las lagunas de conocimiento puede tener implicaciones importantes en las intervenciones educativas, ya que las creencias erróneas pueden ser más persistentes y resistentes al cambio, mientras que las lagunas de información pueden ser abordadas con una mayor facilidad proporcionando los resultados de investigación a los maestros. En nuestro caso, los maestros tenían alrededor de un $35 \%$ de lagunas de conocimiento (PSE $=35.3 \%$ y $\mathrm{PCE}=34.5$ $\%$, ver Tabla 3 para ítems específicos). Cuando un maestro no tiene un conocimiento, hay que proporcionárselo en los programas formativos. En este sentido, algunos estudios de formación de profesores (Aguiar et al., 2014; Nussey, Pistrang, \& Murphy, 2013) han demostrado cómo al proporcionar información científica acerca del TDAH, los profesores la aceptan. Sin embargo, como hemos visto, el hecho de que algunas de las creencias erróneas de los maestros se encuentren en diferentes estudios podría sugerir que se resisten al cambio, y que hay que abordarlas específicamente en los programas de formación. En nuestro estudio, alrededor del $10 \%$ de los maestros tenían creencias erróneas sobre el total de la escala (PSE $=7.2 \%$ y $\mathrm{PCE}=10.3$ $\%$, ver Tabla 3 para ítems específicos). En este sentido, de acuerdo con nuestro conocimiento, solo el estudio de Barbaresi y Olsen (1998) ha analizado específicamente la disminución de las creencias erróneas de los maestros de primaria acerca del TDAH, tras la participación en un programa de la prestigiosa asociación americana Chadd, que dura tan solo dos horas y media. Así, a pesar de una formación de tan corta duración, los maestros disminuyeron sus creencias erróneas acerca de la pobre educación familiar como origen del TDAH (pasó de 41 al $7 \%$ ), también disminuyó la creencia de que las dietas alimenticias eran útiles en el TDAH (pasó del $41 \%$ al $5 \%$ ). Aunque estos resultados no logran erradicar totalmente las creencias erróneas, destacan la posibilidad de hacerlo con el diseño de programas formativos que aborden de forma específica dichas creencias erróneas dentro de los contenidos de los programas formativos.

\section{Referencias}

Aguiar, A. P., Kieling, R. R., Costa, A. C., Chardosim, N., Dorneles, B. V., Almeida, M. R., ...Rohde, L. A. (2014). Increasing teachers' knowledge about ADHD and learning disorders: An investigation on the role of a psychoeducational intervention. Journal of attention disorders, 18 (8), 691-698.

Akram, G., Thomson, A. H., Boyter, A. C., \& McLarty, M. (2009). ADHD and the role of medication: Knowledge and perceptions of qualified and student teachers. European Journal of Special Needs Education, 24(4), 423-436. http://doi.org/10.1080/08856250 903223088

Alkahtani, K. D. F. (2013). Teachers' knowledge and misconceptions of attention deficit/ hyperactivity disorder. Psychology, 4(12), 963-969. http://doi.org/10.4236/psych.201 3.412139

American Psychiatric Association. (2014). Manual Diagnóstico y Estadístico de los Trastornos Mentales. DSM5 (5 $5^{\mathrm{a}}$ ed.). Madrid: Autor.

Anderson, D. L., Watt, S. E., \& Shanley, D. C. (2017). Ambivalent attitudes about teaching children with attention deficit hyperactivity diosrder (ADHD). Emotional and Behavioral Difficulties, 22(4), 332-349. http://doi.org/10.1080/13632752. 2017.1298242

Anderson, D. L., Watt, S. E., Noble, W., \& Shanley, D. C. (2012). Knowledge of attention deficit hyperactivity disorder (ADHD) and attitudes toward teaching children with ADHD: The role of teaching experience. Psychology in the Schools, 49(6), 511-525. https://doi.org/10.1002/pits.2161 7

Barbaresi, W., \& Olsen, R. (1998). An ADHD educational intervention for elementary schoolteachers: A pilot study. 
Developmental and Behavioral Pediatrics, 19(2), 94-100. http://doi.org/10.1097/0000 4703-199804000-00006

Bekle, B. (2004). Knowledge and attitudes about Attention-Deficit Hyperactivity Disorder (ADHD): A comparison between practicing teachers and undergraduate education students. Journal of Attention Disorders, 7(3), 151-161. http://doi.org/10. 1177/108705470400700303

Blotnicky-Gallant, P., Martin, C., McGonnell, M., \& Corkum, P. (2015). Nova Scotia teachers' ADHD knowledge, beliefs, and classroom management practices. Canadian Journal of School Psychology, 30(1), 3-21. ht tp://doi.org/10.1177/0829573514542225

Brook, U., Watemberg, N., \& Geva, D. (2000). Attitude and knowledge of attention deficit hyperactivity disorder and learning disability among high school teachers. Patient Education and Counseling, 40(3), 247-252. http://doi.org/10.1016/S0738-399 1(99)00080-4

Canals, J., Morales-Hidalgo, P., Jané, M. C., \& Domènech, E. (2018). ADHD prevalence in Spanish Preschoolers: Comorbidity, Socio-demographic Factors, and Functional Consequences. Journal of Attentional Disorders, 22(2), 143-153. http: //doi.org/10.1177/1087054716638511

Canu, W., \& Mancil, E. (2012). An examination of teacher trainees' knowledge of attentiondeficit/hyperactivity disorder. School Mental Health, 4(2), 105-114. http://doi.org/10.10 07/s12310-012-9071-3

Catalá-López, F., Peiró, S., Ridao, M., SanfelixGimeno, G., Génova-Maleras, R., \& Catalá, M. A. (2012). Prevalence of attention deficit hyperactivity disorder among children and adolescents in Spain: a systematic review and meta-analysis of epidemiological studies. BMC Psychiatry, 12, 1-13.

Choi, Y. S. (2017). Assessment of Elementary Teachers' Knowledge about ADHD, Attitude, Stress Level and intention to Inclusion Education. Journal of Digital
Convergence, 15(5), 363-372. http://doi.org /10.14400/JDC.2017.15.5.363

Ghanizadeh, A., Bahredar, M., \& Moeini, S. (2006). Knowledge and attitudes towards attention deficit hyperactivity disorder among elementary school teachers. Patient Education and Counselling, 63(1-2), 84-88. $\mathrm{h}$ ttp://doi.org/10.1016/j.pec.2005.09.002

Graczyk, P. A., Atkins, M. S., Jackson, M. M., Letendre, J. A., Kim-Cohen, J., Baumann, B. L., \& McCoy, J. (2005). Urban educators' perceptions of interventions for students with Attention Deficit Hyperactivity Disorder: A preliminary investigation. Behavioral Disorders, 30(2), 95-104.

Graeper, K. D., Barker, K. A., \& Terjesen, M. D. (2008, mayo). Knowledge of ADHD among Vietnamese and American Teachers. Collaborative Research Journal of School Psychology, 18-23.

Greene, R. W., Abidin, R. R., \& Kmetz, C. (1997). The Index of Teaching Stress: A measure of student-teacher compatibility. Journal of School Psychology, 35 (3), 239-259.

Guerra, F. R., \& Brown, M. S. (2012). Teacher knowledge of Attention Deficit Hyperactivity Disorder among middle school students in South Texas. Research in Middle Level Education, 36(3), 1-7.

Havey, J. M. (2007). A comparison of Dutch and US teachers' perceptions of the incidence and management of ADHD. School Psychology International, 28(1), 46-52. http: //doi.org/10.1177/0143034307075679

Hepperlen, T. M., Clay, D. L., Henly, G. A., \& Barké, C. R. (2002). Measuring teacher attitudes and expectations toward students with ADHD: Development of the test of knowledge about ADHD (KADD). Journal of Attention Disorders, 5(3), 133-142. http:/ /doi.org/10.1177/108705470200500301

Hinshaw, S. P. (2018). Attention Deficit Hyperactivity Disorder (ADHD): Controversy, Developmental Mechanisms, and Multiple Levels of Analysis. Annual Review of Clinical Psychology, 14, 291-316. http://doi.org/10.1146/annurev-cl inpsy-050817-084917 
Holst, J. (2008). Danish teachers' conception of challenging behaviour and DAMP/ADHD. Early child development and care, 178(4), 363-374. https://doi.org/10.1080/03004430 701321621

Hong, Y. (2008). Teachers' perceptions of young children with ADHD in Korea. Early Child Development and Care, 178(4), 399-414. ht tp://doi.org/10.1080/03004430701321829

Jarque, S., \& Tárraga, R. (2009). Comparación de los conocimientos sobre el trastorno por déficit de atención con hiperactividad (TDAH) de los maestros en activo y los futuros educadores. Infancia y Aprendizaje, $32(4), 517-529$.

Jarque, S., Tárraga, R., \& Miranda, A. (2007). Conocimientos, concepciones erróneas y lagunas de los maestros sobre el trastorno por déficit de atención con hiperactividad. Psicothema, 19(4), 585-590.

Jerome, L., Gordon, M., \& Hustler, P. (1994). A comparison of American and Canadian teachers' knowledge and attitudes towards Attention Deficit Hyperactivity Disorder (ADHD). Canadian Journal of Psychiatry, 39(9), 563-567.

Jerome, L., Washington, P., Laine, C. J., \& Segal, A. (1999). Graduating teachers' knowledge and attitudes about attention-deficit/ hyperactivity disorder: A comparison with practicing teachers. The Canadian Journal of Psychiatry, 44(2), 192.

Kos, J., Richdale, A., \& Jackson, S. (2004). Knowledge about attention deficit hyperactivity disorder: A comparison of inservice and pre-service teachers. Psychology in the Schools, 41 (5), 517-526. http://doi.or $\mathrm{g} / 10.1002 /$ pits. 10178

Lawrence, K., Estrada, R. D., \& McCormick, J. (2018). Teachers' experiences with and perceptions of students with attention déficit/hyperactivity disorder. Journal of Pediatric Nursing, 36, 141-148. http://doi.or g/10.1016/J.PEDN.2017.06.010

Norvilitis, J. M., \& Fang, P. (2005). Perceptions of ADHD in China and the United States: A preliminary study. Journal of Attention
Disorders, 9(2), 413-424. http://doi.org/10. 1177/1087054705281123

Nur, N., \& Kavakci, O. (2010). Elementary school teachers' knowledge and attitudes related to attention deficit hyperactivity disorder. Health MED, 4(2), 350-355. Recuperado de http://www.healthmedjour nal.com/articles.htm

Nussey, C., Pistrang, N., \& Murphy, T. (2013). How does psychoeducation help? A review of the effects of providing information about Tourette syndrome and attentiondeficit/hyperactivity disorder. Child: care, health and development, 39(5), 617-627.

Ohan, J., Cormier, N., Hepp, S., Visser, T., \& Strain, M. (2008). Does knowledge about attention-deficit/ hyperactivity disorder impact teachers' reported behaviors and perceptions? School Psychology Quarterly, 23(3), 436-449.

Perold, M., Louw, C., \& Kleynhans, S. (2010). Primary school teachers' knowledge and misperceptions of attention deficit hyperactivity disorder (ADHD). South African Journal of Education, 30(3), 457-473.

Piccolo-Torsky, J., \& Waishwell, L. (1998). Teachers' knowledge and attitudes regarding attention deficit disorder. ERS Spectrum, 16(1), 36-40.

Polanczyk, G. V., Salum, G. A., Sugaya, L.S., Caye, A., \& Rohde, L. A. (2015). Annual Research Review: A meta-analysis of the worldwide prevalence of mental disorders in children and adolescents. Journal of Child Psychology and Psychiatry, 56(3), 345-365. h ttp://doi.org/10.1111/jcpp.12381

Poznanski, B., Hart, K., \& Cramer, E. D. (2018). Are Teachers Ready? Preservice Teacher Knowledge of Classroom Management and ADHD. School Mental Health, 10(1), 1-13. https://doi.org/10.1007/s12310-018-9 259-2

Raggi, V., Evans, S. W., Hackethorn, B., \& Thompson, J. (2003, junio). Diagnostic efficiency of the Impairment Rating Scale. Ponencia presentada en ISRCAP: 11th Scientific Meeting. Sydney, Australia. 
Rogers, M., \& Tannock, R. (2018). Are Classrooms meeting the basic psychological needs of children with ADHD symptoms? A self-determination theory perspective. Journal of Attentional Disorders, 22(14), 1354-1360. http://doi.org/10.1177/108705 4713508926

Sciutto, M. J., Terjesen, M. D., \& Bender Frank, A. S. (2000). Teachers' knowledge and misperceptions of attention-deficit/ hyperactivity disorder. Psychology in the Schools, 37(2), 115-122.

Sciutto, M. J., Terjesen, M. D., Kučerová, A., Michalová, Z., Schmiedeler, S., Antonopoulou, K., ...Rossouw, J. (2016). Cross-National Comparisons of Teachers' Knowledge and Misconceptions of ADHD. International Perspectives in Psychology: Research, Practice, Consultation, 5(1), 34-50. http://doi.org/10.1037/ipp0000045

Skaalvik, E. M., \& Skaalvik, S. (2009). Does school context matter? Relations with teacher burnout and job satisfaction. Teaching and Teacher Education, 25(3), 518-524

Snider, V., Busch, T., \& Arrowood, L. (2003). Teacher knowledge of stimulant medication and ADHD. Remedial and Special Education, 24(1), 46-56.

Soroa, M., Gorostiaga, A., \& Balluerka, N. (2016). Conocimiento de los docentes sobre el TDAH: relevancia de la formación y de las percepciones individuales. Revista de Psicodidáctica, 21 (2), 205-226. http://doi .org/10.1387/RevPsicodidact.14023

Syed, E. K., \& Hussein, S. A. (2010). Increase in Teachers' Knowledge About ADHD After a Week-Long Training Program, A Pilot Study. Journal of Attention Disorders, 13(4), 420-423. http://doi.org/10.1177/10870547 08329972

Toye, M. K., Wilson, C., \& Wardle, G. A. (2019). Education professionals' attitudes towards the inclusion of children with ADHD: the role of knowledge and stigma. Journal of Research in Special Educational Nedds, 19(3), 184-196. http://doi.org/10.1111/1471-3802 .12441
West, J., Taylor, M., Houghton, S., \& Hudyma, S. (2005). A comparison of teachers' and parents' knowledge and beliefs about attention deficit/hyperactivity disorder (ADHD). School Psychology International, 26(2), 192-208. http://doi.org/10.1177/014 3034305052913

Weyandt, L. L., Fulton, K. M., Schepman, S. B., Verdi, G. R., \& Wilson, K. G. (2009). Assessment of teacher and school psychologist knowledge of attentiondeficit/ hyperactivity disorder. Psychology in the Schools, 46(10), 951-960. http://doi.org/10. 1002/pits.20436

\section{Notas}

* Artículo de investigación. 